\title{
The role of bone marrow aspirate cells in the management of atrophic mandibular fractures by mini-invasive surgical approach: Single-institution experience ${ }^{\text {it }}$
}

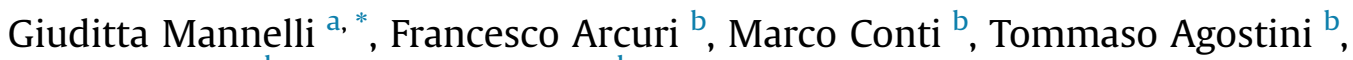 \\ Mirco Raffaini ${ }^{\mathrm{b}}$, Giuseppe Spinelli ${ }^{\mathrm{b}}$ \\ ${ }^{a}$ First Clinic of Otorhinolaryngology Head and Neck Surgery, Department of Surgery and Translational Medicine, University of Florence, Azienda \\ Ospedaliero-Universitaria Careggi, Florence, Italy \\ b Maxillo-Facial Surgery Unit, Neurosensorial Department (Head in Chief: Dr. G. Spinelli), Azienda Ospedaliero-Universitaria Careggi, Florence, Italy
}

\section{A R T I C L E I N F O}

\section{Article history:}

Paper received 12 April 2016

Accepted 5 January 2017

Available online 12 January 2017

\section{Keywords:}

Atrophic mandibular fractures Bone graft

Elderly patients management

Bone marrow aspirate cells

Min-invasive surgical approach

\begin{abstract}
A B S T R A C T
Purpose: The treatment of fractures involves addressing the biology of fracture repair and the mechanical stability of fracture fixation. Traditionally it has included the addition of bone graft to enhance healing. New advances in the understanding of the cellular and molecular mechanisms of fracture repair have led to the use of growth factors to accelerate bone healing. This study aimed to assess the advantages of autologous stem cell use for atrophic mandibular fracture treatment in comparison to standard technique.

Materials and methods: A total of 35 patients (14 male and 21 female) were treated for fractures of atrophic mandibles between January 2011 and December 2014. Surgical technique provided miniinvasive open reduction with or without immediate homologous bone graft, while selected patients received autologous bone marrow aspirate cell grafts in addition to the standard treatment. Demographic data and details of treatment and outcomes were recorded.

Results: Patients were categorized according to the use of autologous stem cells, leaving 17 patients treated with standard technique and autologous stem cells (Group A) and 18 treated with standard osteosynthesis only (Group B). Of the 35 patients, 26 had bilateral fractures; most patients had significant medical co-morbidities. Immediate bone graft was used in 37.1\% of patients ( 7 in group A and 6 in group B). Complications occurred in 5 patients (14.3\%). Two patients in group B (11.1\%) showed non-union of the fracture. One patient in group A (5.8\%) and one patient in group B (5.5\%) showed wound dehiscence and were treated conservatively; one patient in group B had a local infection (5.5\%), one out of 35 (2.8\%), that was managed by prolonged antibiotic treatment.

Conclusions: Despite the advanced age and medical co-morbidities of the vast majority of patients, miniinvasive open approach with autologous bone graft ensures a fast and excellent recovery. Moreover, the management of atrophic mandibular fractures by bone marrow aspirate cells is a safe and useful procedure which has a lower complication rate when compared to standard technique.
\end{abstract}

@ 2017 European Association for Cranio-Maxillo-Facial Surgery. Published by Elsevier Ltd. All rights reserved.

\footnotetext{
Preliminary results of this paper were presented at the European Association for Cranio Maxillo-Facial Surgery meeting held in London in September, 13-16, 2016, as Oral Presentation.

* Corresponding author. First Clinic of Otorhinolaryngology Head and Neck Surgery, Department of Surgery and Translational Medicine, University of Florence, Azienda Ospedaliero-Universitaria Careggi, Via Largo Brambilla 3, 50134 Florence, Italy.

E-mail address: mannelli.giuditta@gmail.com (G. Mannelli).
}

\section{Introduction}

Atrophy can be considered the end stage of edentulism; it leads to a decrease in bone mandibular mass, thus making it more vulnerable to fracture. In 1996, mandibular atrophy was classified by Luhr et al. (1996) into three categories based on mandibular height: Class I, 16-20 mm; Class II, 11-15 mm; and Class III, $<10 \mathrm{~mm}$. Today, about the $10 \%$ of population of developed countries is completely edentulous. Despite the total tooth loss process, the 
rate has fallen over the last decades, thanks to medical progress; however, it is estimated that percentage of edentulous individuals over age 65 years will remain constant in the future, because of the increasing elderly population. Edentulism is more prevalent in persons with less than a high school education, those without dental care history, heavy smokers and alcohol abusers. The percentage of edentulous population varies by countries; today, approximately $8 \%$ of the adults in the United States are completely edentulous (Beltrán-Aguilar et al., 2007). The percentage ranges between $8 \%$ and $15 \%$ in Europe, with a value of about $10.5 \%$ in Italy, together with a consistent difference between the northern and southern regions of the country. In Italy, about $22.6 \%$ of people between 65 and 69 years of age are edentulous; the percentage rises to $34.8 \%$ between 70 and 74 years of age, is almost doubled between 75 and 79 years, and reaches $60 \%$ in people more than 80 years of age.

Because fractures of the edentulous mandible are relatively uncommon, most practitioners have limited experience in treating patients with this problem. Treatment of fractures in older patients with bone atrophy is characterized by high morbidity due to local and general factors (Ellis and Price, 2008). In fact, serious complications are common, especially in patients with atrophy classes II and III, such as non-union or fracture of hardware, the incidence of which has been reported to range between $4 \%$ and 20\% (Luhr et al., 1996; Bruce and Strachan, 1976; Buchbinder, 1993; Eyrich et al., 1997; Iatrou et al., 1998; Kunz et al., 2001) and is probably linked to the bone height in the area of fractures (Bruce and Ellis, 1993; Wittwer et al., 2006).

Another issue related to atrophic mandibular fractures is the comorbidities that are often exhibited by aged patients, which can increase the risk of general anesthesia (Yamamoto et al., 2011; Clayman and Rossi, 2012). In addition, the physiologically decreased osteogenesis process, together with local factors relating to atrophic cortical bone and inadequate blood supply, contribute to make this surgery challenging to physicians (Wongchuensoontorn et al., 2009).

The basic principles of atrophic mandible fracture management include open reduction and internal fixation to achieve restoration in terms of aesthetics and functionality. The surgical technique has evolved through decades, passing from the use of intraosseous wires to fix fractures in the 1970s (Bruce and Strachan, 1976), by the introduction of internal fixation method by osteosynthesis plates and screws in the 1990s, together with the first report discouraging the clinical and biological value of bone grafting in older patients because of their high number of co-morbidities (Bruce and Ellis, 1993). However, in 2008, Ellis and Price (2008) showed that atrophic mandibular fractures treated by open reduction, internal plate fixation and immediate bone grafts had an extremely high rate of success. Although they encountered no problems from taking autogenous grafts in their patients, harvesting a bone graft from an elderly patient could be problematic. Autografts can come from a variety of areas. The iliac crest is by the far the most common source, due to the rich presence of progenitor cells and growth factors, limited donor site morbidity, fair bone quantity and relative ease of harvest. It would therefore be preferable to provide osteogenic potential in a less invasive manner. Recently, the concept of tissue engineering and regenerative medicine has been widely accepted in medicine (Langer and Vacanti, 1993), and several clinical studies have been performed in the field of oral and maxillofacial surgery, especially concerning oral rehabilitation, including studies of bone and periodontal regenerative medicine. In fact, the vast majority of articles published recently in the literature have focused on the use of autologous stem cells in atrophic mandibles to induce and enhance new bone formation to allow implant placement. For instance, Rickert et al. (2011), Katagiri et al. (2016),
Kawai et al. (2015), and Yamada et al. (2013) have observed and documented the safe use of mesenchymal stem cell (MSC) bone marrow (BM)-derived bone augmentation for dental implants in patients affected by severe alveolar bone atrophy. All of these authors indicated that this minimally invasive bone regeneration technique was a useful and novel option in dental implant treatment of severe bone atrophy, showing good results through MSCs' great osteogenic potential, together with poor donor site morbidity and few inflammatory signs. In support of these statements, other reports of animal models have presented encouraging results. Osugi et al. (2012) have shown how MSCs enhance migration, proliferation and osteogenic marker expression in a rat calvarian bone defect model, and this was confirmed by Inukai et al. (2013) a few months later. An interesting article stating the capacity of MSCs to regulate the turnover of local bone by a paracrine effect, already mentioned by Herford and Boyne (2008), was reported in a rat bisphosphonate-related osteonecrosis of the jaw-like model by Ogata et al., 2015 , for which histological analysis confirmed the presence of osteoclasts in the MSC group.

However, clinical use of stem cells requires highly qualified safety investigation and quality management of cell handling, and it is very expensive. These limitations currently impede the widespread use of stem cells for alveolar bone regeneration therapy; moreover, it has been shown that the implanted cells do not survive long (Muller-Ehmsen et al., 2002; Toma et al., 2009; Ide et al., 2010). It is known that osteoblasts develop from the proliferation and differentiation of osteoprogenitor cells and that these progenitors arise from a bone marrow (BM) population called mesenchymal stem cells (MSCs). MSCs have the ability to self-renew and present a multilineage potential (Kan et al., 2005). Their plasticity makes them a promising resource for research in cellular therapy of tissue defects, and several authors have demonstrated how autologous stem cells aspirated from BM can enhance bone healing in in vitro and in vivo studies (Kan et al., 2005; Rickert et al., 2011; Watson et al., 2014; Katagiri et al., 2013; Osugi et al., 2012; Inukai et al., 2013; Kawai et al., 2015; Katagiri et al., 2016). In this regard, osteoinductive growth factors such as bone morphogenic protein (BMP)-2 have been already used with osteoconductive materials to promote bone regeneration (Herford and Boyne, 2008). For example, platelet-rich plasma (PRP), collected by centrifugation of autologous peripheral blood, has been reported to enhance bone regeneration or to promote soft and hard tissue healing (Yamada et al., 2013). PRP includes growth factors such as platelet-derived growth factor (PDGF), transforming growth factor- $\beta$ (TGF- $\beta$ ), vascular endothelial growth factor (VEGF) and epithelial growth factor (EGF) (Marx et al., 1988). PRP also has several advantages over the use of animal sera or recombinant factors from potentially safe and effective clinical applications (Prins et al., 2009).

In this scenario, we present our view of atrophic mandibular fracture management, by presenting a consistent single surgeon case series. We review this series to confirm the usefulness of a minimally invasive surgical approach to the treatment of this type of fracture, and to assess the great regenerative potential of autologous stem cells grafted from the anterior iliac bone to facilitate bone healing and as well as recovery in patients with mandibular moderate-to-severe atrophy. Our data respect the epidemiology of this pathology, which is linked to the fact that our geographic area, Tuscany, represents the region with one of the highest concentration of senior citizens in Italy, with a global percentage of adults more than 65 years old of $23.2 \%$, against a national value of $20.2 \%$; most of them are females (58\%), and 62\% are over 75 years, in comparison to a national rate of women over 75 of $52 \%$. Therefore, our tertiary care hospital represents the reference center for this type of uncommon fractures, justified by the presence of a large number of elderly patients. 


\section{Materials and methods}

\subsection{Study population}

This prospective study included 35 patients treated by open reduction, with or without osteogenic BMAC use, of fractures of completely edentulous atrophic mandibles ( $\leq 15 \mathrm{~mm}$ bone height in the area of fracture) at the Maxillo-Facial Surgery Unit of Florence between January 2011 and December 2014. Only patients over 65 years of age were enrolled in our study, because it represents the elderly population, who are the main individuals affected by severe mandible atrophy, the challenging management of which is influenced by a high prevalence of co-morbidities in comparison to those in younger patients. On the other hand, fractures of atrophic mandible class I were excluded because we wanted to focus our attention on the most difficult cases only, considering that serious complications are commonly reported for class II and III in up to $20 \%$ of cases. Moreover, patients not meeting these inclusion terms, patients presenting with pathological fractures, patients who did not give their own consent to be enrolled in this study protocol, patients with a previous medical history of mandible fractures, patients who had undergone head and neck radiotherapy treatment, and patients with insufficient preoperative or postoperative data were excluded from our analysis. Patients were given extensive information about the procedures, including the surgery, graft material, plates, and screws and the uncertainties of using a new bone-regenerative method.

The research protocol was approved by the Institute's and University Ethics Committee on human research and was conducted in accordance with all accepted standards for human clinical research. All patients who accepted enrollment in this study gave their informed consent.

The following information was collected from the patients' medical records: age and sex, concomitant systemic medical conditions, cause and site(s) of fracture(s), height of bone in the area of fracture(s), displacement of fracture(s), surgical details, adequacy of reduction, postoperative pain and diffuse local hematoma, hospital length of stay, long-term complications and duration of follow-up.

A preoperative panoramic radiograph was used to evaluate the height of bone in the area of fracture, the value of which is always given to us by radiologists. The amount of displacement of each fracture was grossly graded by us from mild to severe, where mild displacement represented a fracture with a slight separation or angulation of segments, moderate displacement showed the presence of a significant displacement of fragments, and severe displacement showed that mandibular segments were not all aligned with one another with gross override (Ellis and Price, 2008) (Fig. 1).

To better assess the surgical advantages of our approach and to compare the incidence of postoperative complications, patients were prospectively randomly categorized into two treatment groups. Group A was treated with a more conservative approach, based on small skin incisions less than $4.5 \mathrm{~cm}$ in length, with the use of standard osteosynthesis plates and screws of $2.0 \mathrm{~mm}$, even in case of comminuted and displaced fractures, plus the addition of autologous Bone Marrow Aspirate Concentrates (BMACs). Group B was treated with a more aggressive surgical approach, with extended mandibular bone exposure together the use of large reconstructive osteosynthesis plates in case of severe comminuted and displaced mandibular fractures.

In both groups, bone grafts were used in all cases of comminuted and displaced fractures. Reconstructive osteosynthesis plates were avoided for patients in group A, and BMACs were used only in group A. Fig. 2 provides a patient flow chart.

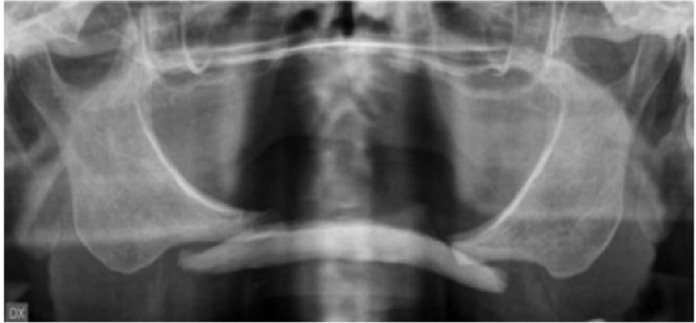

Fig. 1. Radiograph representing a displaced bilateral body mandibular fracture.

\subsection{Surgical technique}

Surgery was performed within a maximum of 6 days from the trauma in all patients. All surgical procedures were performed by the senior surgeon (G.S.).

Surgical details tabulated included a surgical approach (minimally invasive versus aggressive), presence of comminution or defect (yes or no), method of reduction of fragments (forceps, bone clamps, bone plates, wires), simultaneous bone grafting (yes or no), use of drains (yes or no), surgical procedure length, and intraoperative complications. The reduction was judged to be good if the postoperative radiograph showed a continuous alignments of fragments, and was considered to be poor if the radiograph showed a discontinuity in alignment, such as a break of flaring of a ramus on the posteroanterior radiograph.

Group A provided the following surgical procedures: $2.0-\mathrm{mm}$ titanium locking "reconstruction" plates with a minimum of 3 screws on each side of the fracture in all cases. A minimally invasive skin incision approach was used in all cases, with an open access less than $4.5 \mathrm{~cm}$ length (Fig. 3a-e). This aimed to achieve the lowest mandibular bone exposure, in order to reduce possible late complications such as wound dehiscence, local infection, and plate extrusion.

Group B received large reconstructive osteosynthesis plates with screws $2.3 \mathrm{~mm}$ in case of severe comminuted and displaced mandibular fractures, with a large skin access (Fig. 4). All other cases provided screws $2.0 \mathrm{~mm}$ but still through a skin access larger than $4.5 \mathrm{~cm}$ in length.

Osteosynthesis plates were always placed extra-periosteally in both groups, and allo-bone bank grafts were used in all cases of comminuted and displaced fractures.

Suction and/or Penrose drains were used in 7 patients, all of whom had bone grafts placed at the time of surgery. The drains were placed below the platysma muscle and were removed on either the next day $(n=5)$ or day after that $(n=2)$. None of selected patients received autologous bone grafts, in order to achieve the lowest incidence of surgical morbidity in such challenging and already debilitated patients.

\subsection{Bone marrow cell preparation}

To harvest the bone marrow cells, the iliac crest bone was punctured with a bone marrow biopsy needle (Bone Marrow Aspiration Pack, Harvest Technologies Corporation, Plymouth, MA, USA) (Fig. 5). A 32-ml quantity of bone marrow was collected and the aspirate was then transferred quickly into 4 RegenTHT tubes (RengenKit Extracell BMC:Ref:R-EXB/T//Ref:R-EXB/T2). Each syringe containing the medullary blood was connected to the transfer device and put it on the stopper of RegenTHT by carefully applying a light pressure; in this way the blood was drawn up directly by the tube (final volume about 5-8 ml). Then a self-adhesive disc was 


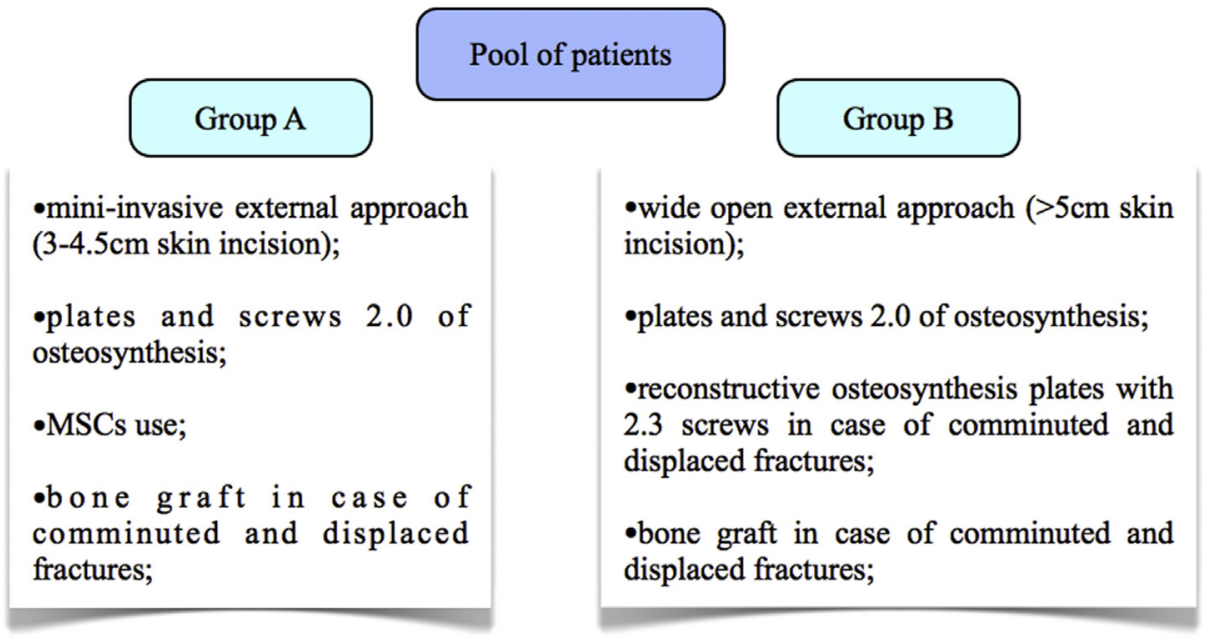

Fig. 2. Flow chart of patients recruited for the study and different surgical treatment protocols.
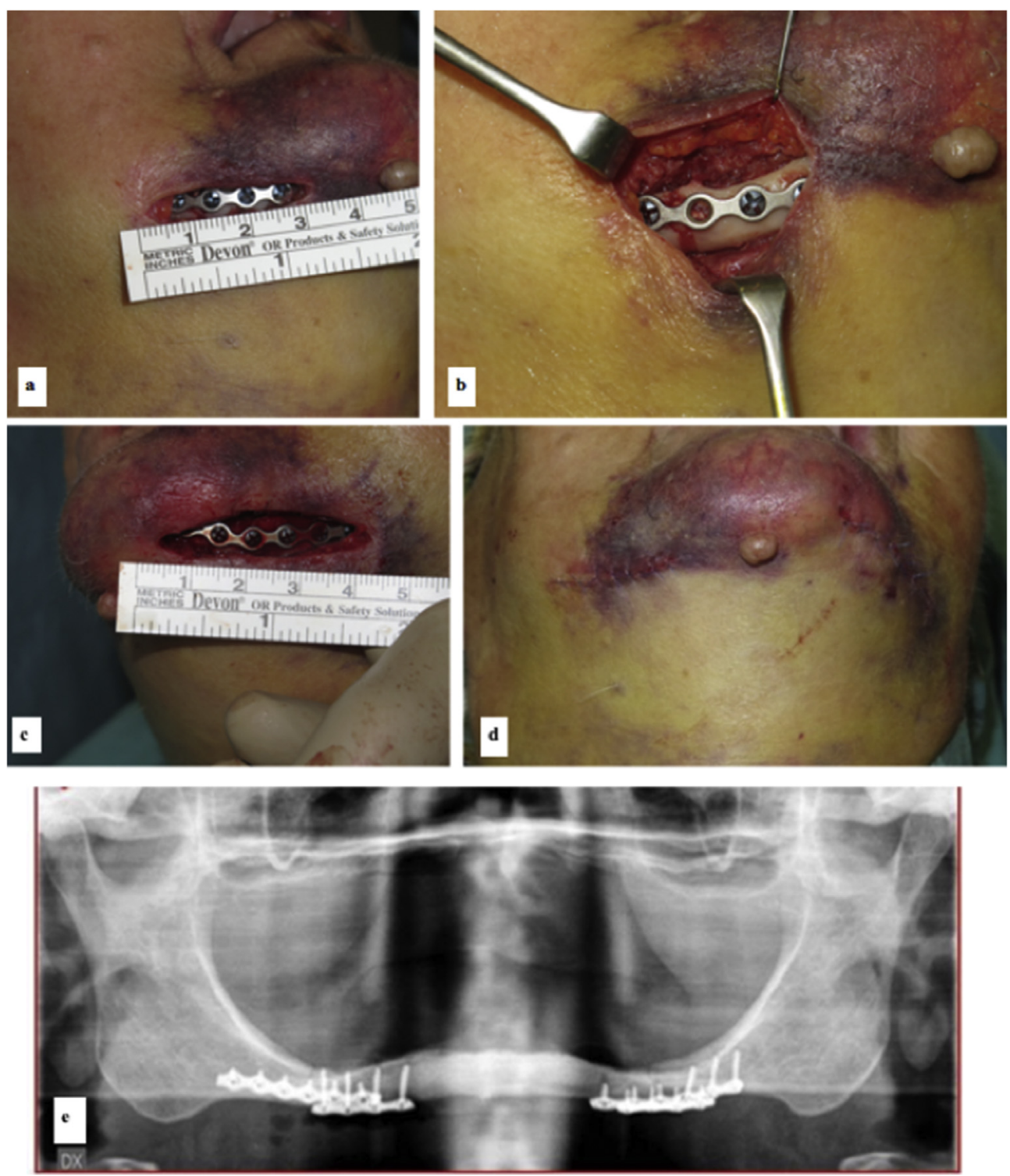

Fig. 3. Minimally invasive surgical approach provided by group A protocol: a) minimally invasive bilateral open access with skin incision length of less than 4.5 mm on right side; b) use of plates and screws $2.0 ; \mathrm{c}$ ) and on left side; d) intraoperative final results; e) postoperative radiograph showing plates and screws 2.0 used for fracture reduction. 


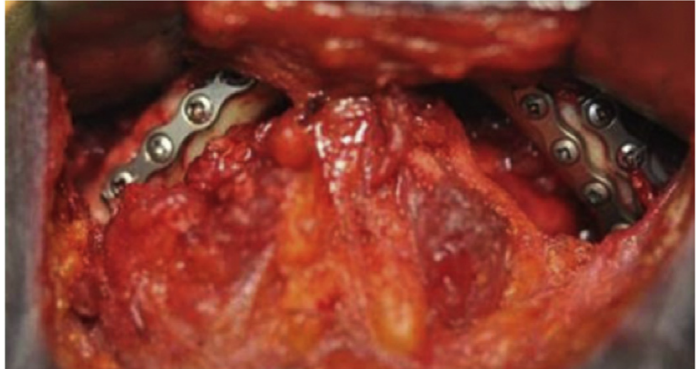

Fig. 4. Aggressive surgical approach in accordance with group B protocol: bilateral body displaced fracture treated by large reconstructive osteosynthesis plate and 2.3 screws through large extra-oral access.

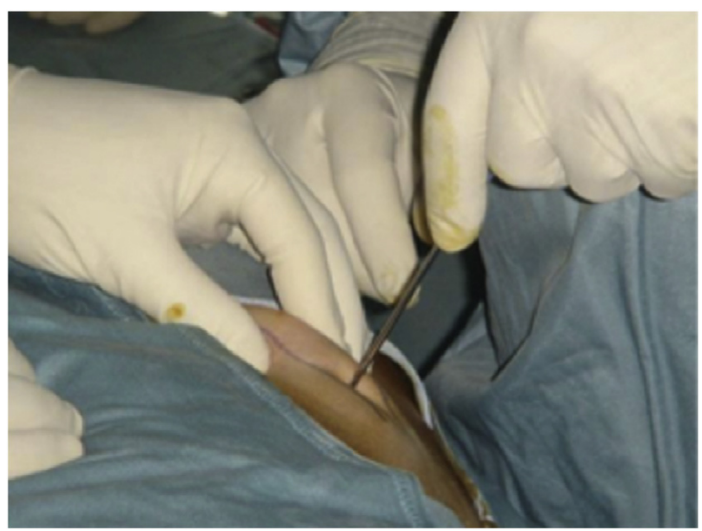

Fig. 5. Bone marrow biopsy puncture in sterile condition. applied to seal the hole in the protective film covering the tube, and the tube was inverted gently to obtain homogeneous diffusion of the anti-coagulant.

Bone marrow cells were isolated using the Bone Marrow Aspirate Concentrate (BMAC) system (Bone Marrow Procedure Pack, Harvest Technologies Corporation, Plymouth, MA, USA) according to the manufacturer's instructions. The system can use up to two disposable containers with two chambers. The first chamber contains a floating shelf of a specific density by which the red blood cells are separated from the nucleated cells, platelets and plasma during the initial centrifugation phase. Cellular elements and plasma are automatically transferred into the second chamber and concentrated by centrifugation. A portion of the supernatant plasma is removed and the cellular elements are resuspended in the remaining plasma. The concentration process was evaluated by Haemocytometry (Sysmex Analyzer, SYSMEX EUROPE GMBH, Norderstedt, Germany). Tubes were centrifuged for 8 min with a force of $1600 \mathrm{~g}$ (according to the centrifuge manufacturer's instructions). Renege THT tubes were inverted gently several times (at least $20 \times$ ) to resuspend the cellular elements into the plasma. Finally, the medullary plasma was withdrawn by using a sterile syringe equipped with a transfer device (see RengenKit Extracell in Fig. 6).

Preoperative hematological assessments included a complete blood count (CBC) with platelet levels. Platelet-rich plasma (PRP) was isolated in a 200-ml collection bag containing the anti-coagulant citrate under sterilized conditions. Briefly, the blood was centrifuged for $10 \mathrm{~min}$ at $1500 \mathrm{rpm}$. Yellow plasma (containing the buffy coat, which included platelets and leukocytes) was obtained and a second centrifugation at $3500 \mathrm{rpm}$ for 5 min was performed; then the plasma supernatant was removed. The resulting pellet of platelets, the buffy coat/plasma fraction (PRP), was resuspended in the residual $20 \mathrm{ml}$ of plasma and used in the platelet gel. The PRP

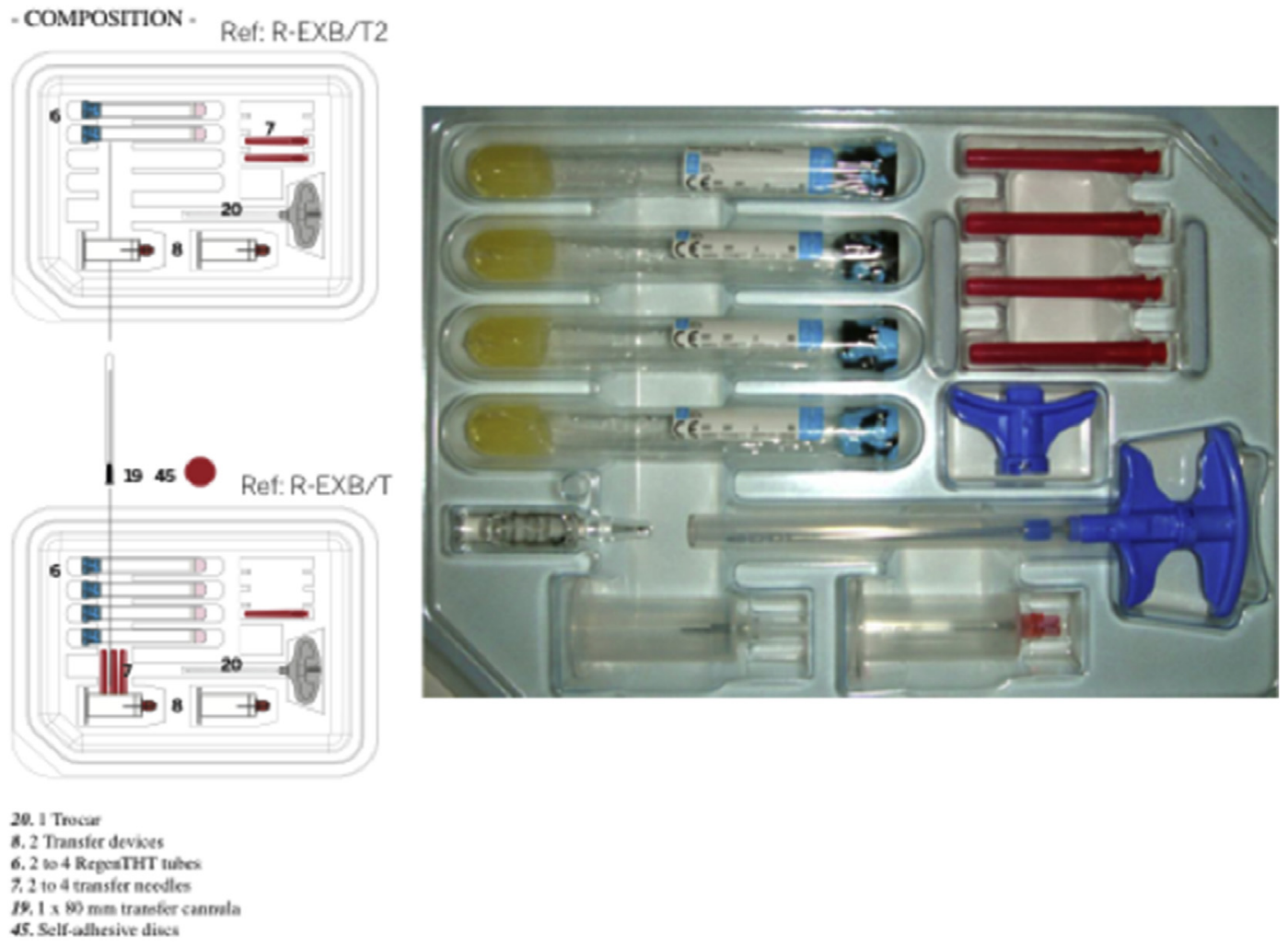

Fig. 6. RengenKit Extracell BMC:Ref:R-EXB/T//Ref:R-EXB/T2. 
platelet count was 994,000. The values confirmed the platelet sequestration ability of the process, which showed that the concentration was $450 \%$ above the baseline platelet count.

Human thrombin in powdered form (5000 units) was dissolved in $5 \mathrm{ml}$ of $10 \%$ calcium chloride in a separate sterile cup.

Finally, PRP, BMACs, and air were aspirated into a sterile syringe. In another syringe, the thrombin/calcium chloride mixture was aspirated. The cells were resuspended directly into the PRP. The two syringes were connected with a T connector, and the plungers of the syringes were alternatively pushed and pulled. The mixing contents (tissue engineered bone, TEB) assumed a gel-like consistency (Fig. 7a) as the thrombin affected the polymerization of the fibrin. The TEB plus PRP was placed under the periosteum once the osteosynthesis plates were positioned and fixed, in order to enhance tissue healing (Fig. 7b).

\subsection{Postoperative follow-up}

Recovery time and hospital length of stay were counted, together with the clinical evaluation of postoperative pain by a visual analogue scale (VAS), where a score between 2 and 4 identified mild pain, between 5 and 7 moderate pain, over 7 severe pain (Fig. 8). Postoperative hematoma was evaluated, but only diffused extended cervical hematoma was considered during the immediate postoperative follow-up, to identify the onset of dysphagia, dyspnea or any other compressive symptoms. This was because, first, the vast majority of our patients were on anti-coagulant medications with a consequent higher risk for bleeding and postoperative hematoma appearance; and second, we were comparing patients affected by the same pathology but treated differently, one group by a more aggressive surgical protocol that might have caused some of the above complications. Clinical evaluation was scheduled at 1 week and at 1, 2, 3, 6 and 12 months after surgery, then annually. Physical assessment together with photographic records and radiographic analysis were routinely performed, and the incidence of any complications such as infection, dehiscence, BMAC donor site problems, loosening and/or fractures of screws/plates were recorded.

Orthopantomography was performed every scheduled visit, and computed tomography (CT) scans were performed at 6 and 12 months after surgery. Radiological investigations were used to assess the quality of surgical reduction, to evaluate bone consolidation and to detect any postoperative complications. Their results were registered in a specific form.

\subsection{Statistical analysis}

Continuous data descriptive statistics were performed, 95\% confidence interval $(\mathrm{CI})$ of the mean and standard deviation were calculated. A $t$-test was used, and $\mathrm{p}<0.05$ was considered statistically significant. Fisher and chi-square tests to analyze a $2 \times 2$ contingency table were used for categorical data.

\section{Results}

\subsection{Study population}

Clinical characteristics are summarized in Tables 1 and 2, including groups A and B data, respectively. Patients' age was almost equivalent among the two groups, in which group A presented with a median age of 77 years (mean $75.47 \pm$ SD $5.23,95 \% \mathrm{CI}$ 72.78-78.16, range 66-85) versus group $B$, whose median age was 76.5 years (mean $75.44 \pm$ SD 5.07, 95\% CI 72.92-77.96, range $66-83)(p=0.9881)$. Of the patients, $31.4 \%$ were male, while $68.9 \%$ were female, with a median age of 74 years (mean $72.82 \pm$ SD 5.47, $95 \% \mathrm{CI} 69.14-76.50$, range $66-85$ ) and 78 years (mean $76.67 \pm$ SD $4.49,95 \%$ CI 74.77-78.56, range 67-83).

The vast majority of patients presented with at least one systemic illness that had to be managed in association with their fractures. The most common co-morbidities were hypertension $(\mathrm{n}=8,25.7 \%)$, chronic obstructive pulmonary disease $(\mathrm{n}=6,17.1 \%)$, osteoporosis ( $\mathrm{n}=4,11.4 \%)$, diabetes $(\mathrm{n}=2,5.7 \%)$, dementia $(\mathrm{n}=2$, $5.7 \%)$ and previous stroke $(\mathrm{n}=2,5.7 \%)$. Only 10 patients had no associated systemic co-morbidities; four of these were the youngest patients.

Mandibular atrophy class III was documented in five patients in group A (14.3\%) and four patients in group B (11.4\%). Causes of fractures included falls $(n=23,65.7 \%)$, vehicular accidents $(n=10$, $28.6 \%)$ and assaults $(\mathrm{n}=2,5.7 \%)$.

The vast majority of cases reported bilateral mandibular fractures (74.3\%), in fact only nine of 35 patients reported a unilateral fracture $(25.7 \%)$, with a final total of 61 mandibular fractures to treat. The nine unilateral fractures were all located in the

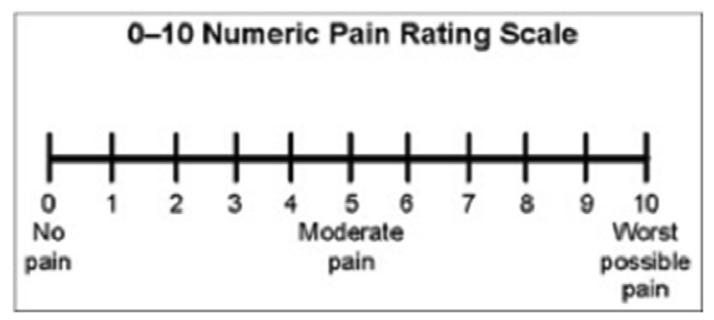

Fig. 8. Visual analogue scale (VAS) for pain: 1) VAS $<2$ denotes no pain, 2) VAS 3-4 denotes mild pain, 3) VAS 5-7 denotes moderate pain, and 4) VAS $>7$ denotes severe pain.
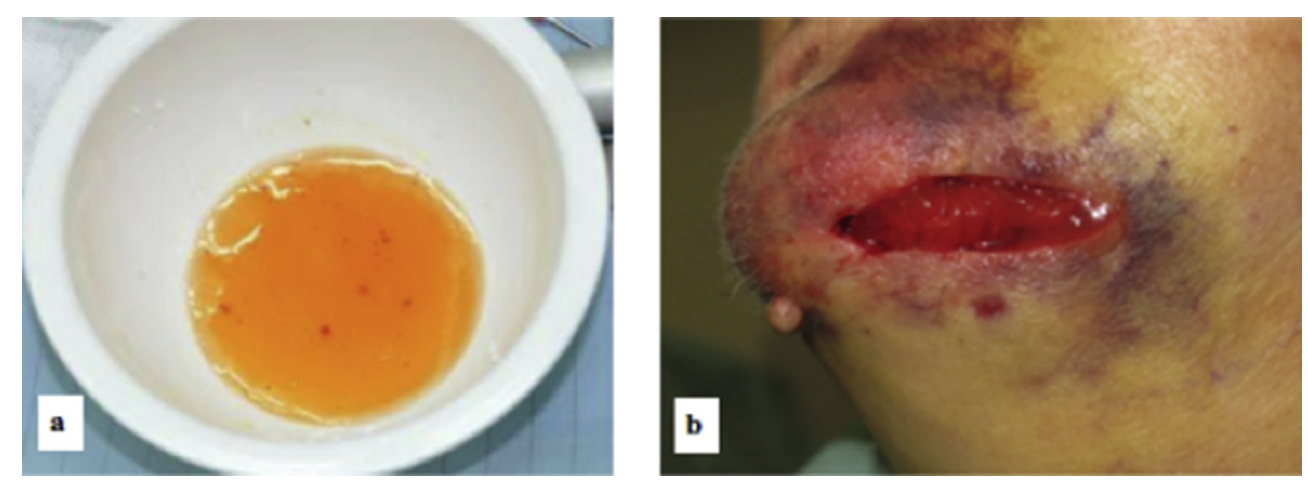

Fig. 7. Platelet-rich plasma (PRP), Bone Marrow Aspirate Concentrate (BMAC) aspirate together the thrombin/calcium chloride mixture resuspended directly into the PRP assumed a gel-like consistency. a) TEB with gel-like consistency; b) TEB positioned sub-periosteally plus PRP dose on osteosynthesis site. TEB = tissue engineered bone. 
Table 1

Characteristics of patients in group A who received BMACs.

\begin{tabular}{|c|c|c|c|c|c|c|c|c|}
\hline Age $(y)$ & Sex & Co-morbidities & Class of atrophy & Type of fracture & Second site of fracture & Comminuted/displaced fracture & Bone graft & Complications \\
\hline 66 & M & None & II & Unilateral & - & Yes & No & None \\
\hline 71 & M & HBP & II & Bilateral & Body region & No & No & None \\
\hline 78 & $\mathrm{~F}$ & HBP & III & Bilateral & Body region & Yes & Yes & None \\
\hline 79 & $\mathrm{~F}$ & HBP & II & Bilateral & Body region & Yes & Yes & None \\
\hline 68 & $\mathrm{~F}$ & None & II & Bilateral & Contralateral angle & Yes & Yes & None \\
\hline 73 & $\mathrm{~F}$ & None & II & Bilateral & Body region & Yes & Yes & None \\
\hline 77 & F & COPD & II & Bilateral & Body region & No & No & None \\
\hline 79 & $\mathrm{~F}$ & HBP & II & Bilateral & Contralateral angle & No & No & None \\
\hline 85 & M & HBP & III & Bilateral & Contralateral angle & Yes & Yes & None \\
\hline 81 & $\mathrm{~F}$ & $\mathrm{DM}$ & III & Unilateral & - & Yes & Yes & Wound dehiscence \\
\hline 77 & $\mathrm{~F}$ & Previous IMA & II & Unilateral & - & Yes & No & None \\
\hline 76 & M & COPD & II & Unilateral & - & No & No & None \\
\hline 75 & M & COPD & III & Bilateral & Contralateral angle & Yes & Yes & None \\
\hline 69 & $\mathrm{~F}$ & OSP & II & Bilateral & Body region & Yes & Yes & None \\
\hline 70 & $\mathrm{~F}$ & None & II & Bilateral & Body region & No & No & None \\
\hline 78 & $\mathrm{~F}$ & COPD & III & Bilateral & Body region & No & No & None \\
\hline 81 & $\mathrm{~F}$ & Dementia & II & Bilateral & Contralateral angle & Yes & Yes & None \\
\hline
\end{tabular}

$\mathrm{M}=$ male; $\mathrm{F}=$ female; $\mathrm{DM}=$ mellitus diabetes; $\mathrm{HBP}=$ high blood pressure; IMA = ischemic myocardial attack; COPD = chronic obstructive pulmonary disease; OSP $=$ osteoporosis

Table 2

Characteristics of patients in group B treated without BMACs.

\begin{tabular}{|c|c|c|c|c|c|c|c|c|}
\hline Age $(y)$ & Sex & Co-morbidities & Class of atrophy & Type of fracture & Second site of fracture & Comminuted/displaced fracture & Bone graft & Complications \\
\hline 69 & M & HBP & II & Unilateral & - & Yes & Yes & None \\
\hline 66 & M & None & II & Unilateral & - & No & No & None \\
\hline 67 & $\mathrm{~F}$ & None & II & Unilateral & - & Yes & No & None \\
\hline 75 & M & Previous IMA & II & Bilateral & Body region & Yes & Yes & Non-union \\
\hline 77 & $\mathrm{~F}$ & OSP & II & Bilateral & Body region & No & No & None \\
\hline 79 & $\mathrm{~F}$ & COPD & II & Bilateral & Body region & No & No & None \\
\hline 80 & $\mathrm{~F}$ & COPD & II & Bilateral & Body region & No & No & None \\
\hline 82 & $\mathrm{~F}$ & OSP & II & Bilateral & Body region & No & No & None \\
\hline 83 & $\mathrm{~F}$ & Dementia & III & Bilateral & Body region & Yes & Yes & Non-union \\
\hline 69 & M & None & II & Bilateral & Body region & No & No & None \\
\hline 74 & M & None & II & Unilateral & - & No & No & None \\
\hline 76 & $\mathrm{~F}$ & OSP & III & Bilateral & Contralateral angle & Yes & Yes & None \\
\hline 72 & $\mathrm{~F}$ & None & II & Unilateral & - & No & No & None \\
\hline 78 & $\mathrm{~F}$ & DM & II & Bilateral & Body region & No & No & Wound dehiscence \\
\hline 75 & M & HBP & II & Bilateral & Body region & Yes & No & Local infection \\
\hline 77 & $\mathrm{~F}$ & HBP & III & Bilateral & Contralateral angle & Yes & Yes & None \\
\hline 80 & $\mathrm{~F}$ & OSP & III & Bilateral & Body region & No & No & None \\
\hline 79 & $\mathrm{~F}$ & None & II & Bilateral & Body region & No & No & None \\
\hline
\end{tabular}

$\mathrm{M}=$ male; $\mathrm{F}=$ female; $\mathrm{DM}=$ mellitus diabetes; $\mathrm{HBP}=$ high blood pressure; $\mathrm{IMA}=$ ischemic myocardial attack; COPD = chronic obstructive pulmonary disease; OSP $=$ osteoporosis

mandibular body; about three-fourths of the bilateral fractures (73.1\%) were diagnosed in the body area, while the remaining $28.9 \%$ presented as second site of fracture the contralateral mandibular angle. Two of the angle fractures were not atrophic $(>15 \mathrm{~mm}$ in height) but nonetheless were included in the analysis for completeness. A total of 18 patients, 11 in group A and 7 in group B, presented with comminuted and displaced fractures (51.4\%), whereas 24 were bilateral and 6 unilateral fractures, totaling 30 overall (49.2\%). Fracture displacement ranged from minimal to severe. Unilateral fractures seemed to be less displaced than bilateral fractures. The most significant displacement occurred in the bilateral body fracture.

A total of 14 patients needed immediate bone graft (40\%); all of them were affected by comminuted and displaced fractures. Nine of 14 patients were treated by a minimally invasive external surgical approach with bone graft plus the use of BMACs (64.3\%) without the use of any reconstructive osteosynthesis plate; the remaining 5 patients underwent operation by a more aggressive surgical approach, in accordance with protocol B, by a more extended skin incision, exposing the whole mandibular body, using the bone graft plus a reconstructive osteosynthesis plate when needed (severe dislocated fractures). Reconstructive osteosynthesis plates were avoided in group $\mathrm{A}$, while they were used in three patients in group B (16.6\%); two reported bilateral comminuted and displaced fractures, and the third with unilateral severe displaced fracture. The overall use of these tools was of $8.6 \%$.

No major intraoperative complications were seen in any patients. However, 5 of 35 patients presented postoperative complications (14.3\%); a total number of 2 non-union fractures were recorded (5.7\%), all of them encountered in group B (2/18, $11.1 \%)$, both presented as bilateral body fractures treated by bone graft, plus reconstructive osteosynthesis plate in case of atrophy class III $(1 / 18,5.5 \%)$. The first case, a man 75 years of age with a mandibular atrophy class II, underwent a surgical revision of the non-union fracture; in contrast, a woman 83 years of age, affected by a mandibular atrophy class III, did not undergo any second surgical procedure, and during her clinical follow-up her functional outcomes remained stable without any worsening of the complication experienced.

A final number of two wound dehiscences were seen (5.7\%), one in group A (1/17, 5.8\%) and one in group B (1/18, 5.5\%), both in patients affected by diabetes and over 70 years of age. Both patients required daily local medications in our clinic for about 3 weeks since they recovered completely. 
Only one patient experienced local infection (2.8\%) in group B $(1 / 18,5.5 \%)$ and he was operated on for a mandibular body bilateral comminuted and displaced fracture treated by a reconstructive osteosynthesis plate. This 75-year-old man had local skin redness, itching and secretions leaking within the second postoperative week. We performed a local wound revision under local anesthesia, and we prescribed systemic antibiotic therapy together with local medications, and a complete wound healing was reached in 3 weeks.

All of our patients showed a steady long-term functional result, together with a complete resolution of local complications, when experienced, within the third month of follow-up; and all of them completed at least 1 year of clinical-radiological control.

\subsection{Surgical data}

Surgery was performed within 6 days from the accident in both groups. The median surgical procedure length was $128 \mathrm{~min}$ in group A, with a mean value of $134 \pm$ SD 5.23 (95\% CI 118.25-149.75, range 95-195) against a median value of 100 min for group B, with a mean value of $105 \pm$ SD 19.45 (95\% CI 95.33-114.67, range 75-141), with a statistically p value of 0.002 . All fractures were treated by open reduction, but the type of osteosynthesis varied among the two groups as explained in Materials and Methods section.

Ten of 17 patients in group A claimed mild to moderate pain during their first postoperative day, and none of them experienced a grade of pain higher than 7 in accordance with the visual pain scale; on the other hand, two patients belonging to group B claimed local severe pain (grade 7) during the postoperative stay, and both reported local wound complications (dehiscence and infection). Only one patient showed extended cervical hematoma in group A, while 5 of 18 patients (27.8\%) presented diffused mandibular and cervical hematoma in group $B(p=0.1774)$.

The median stay at the hospital was 3 days for patients in group A (mean $3.24 \pm$ SD $1.52,95 \%$ CI 2.45-4.02, range 2-7) versus a median stay of 4 days for patients in group B (mean $3.78 \pm$ SD 1.35, $95 \% \mathrm{CI} 3.11-4.45$, range $2-6)(\mathrm{p}=0.2725)$; in the second group, the hospital stay was longer due to the use in two cases of local drain tubes, which have delayed patients discharge of about 2 days; moreover, two other patients belonging to group $\mathrm{B}$ reported local severe pain, thus requiring intravenous injection of analgesic medications for 2 days more than the other patients. Patients who experienced diffuse cervical hematoma were kept longer to assess its resolution.

All data are summarized in Table 3.

\section{Discussion}

There are no prospective, statistically validated, long-term outcome studies concerning the treatment of fractures of the atrophic edentulous mandible. Whether more invasively treated fractures have a greater likelihood of complications and increased general morbidity, and the treatment of choice, are discussed controversially in the literature (Barber, 2001; Marciani, 2001). Surgical techniques have improved considerably since 1976 with Bruce and Strachan (1976), nevertheless, open reduction and internal fixation represent the universal preferred option for atrophic mandibular fractures treatment, so far. Invasive techniques for the treatment of the atrophic mandibular body fracture should be considered when conservative techniques have a high likelihood of non-union. One advantage of open techniques is the possibility of adding autogenous bone grafts, growth factors or cell therapy with minimally increased morbidity.

The advantage of using bone autograft is that there is no risk of disease transmission, and that it is easily available. Although initially it was believed to be a very cost-effective treatment, several studies have demonstrated that the direct and indirect costs of iliac crest bone graft harvest are substantial (St John et al., 2003). Therefore, it is not surprising that recent advancements in regenerative medicine have motivated the progressively more frequent use of engineering tissue in clinical practice to reduce possible donor site co-morbidities and to facilitate complex surgical procedures, and afterwards, it appears to be the best choice in cases of challenging and infrequent patients such as elderly individuals affected by atrophic mandible fractures.

For the time being, however, iliac crest bone graft (ICBG) remains the most affordable source of growth factors for bone stimulation. The main disadvantages of ICBG relate to the limited amount of bone that can be harvested, and the morbidity of the procedure to the patient. Complications such as infection, seroma, hematoma, postoperative pain, scar sensitivity, and meralgia paresthetica have been reported. Less commonly, major complications such as pseudoaneurysm, arteriovenous fistula, pelvic instability, avulsion of the anterior superior iliac spine, and hernia have also been documented. In one study, $2.4 \%$ of patients had major complications, $21.8 \%$ had minor complications, and $37.9 \%$ of patients reported pain localized to the harvest site 6 months postoperatively (Sen and Miclau, 2007). In our study we explained how to achieve a good final result by a less invasive technique, profiting by iliac crest bone as stem cells donor only and using bank bone allograft instead of autologous bone, thus obtaining a reduction of surgical procedure costs and hospital stay, without affecting the final outcome.

In our series, only 3 of 14 bone grafts showed postoperative complications; the one in group A was a 81-year-old woman affected by diabetes and presenting with a unilateral severe displaced mandibular fracture in a class III mandibular atrophy, who, despite the minimally invasive surgical approach together the BMAC use, experienced a wound dehiscence. The other two complications, both non-union fractures, occurred in cases of bilateral comminuted and displaced fractures, one patient presenting with atrophy class III and who received in addition the reconstructive osteosynthesis plate. Despite the fact that the comparison of postoperative complication events between the two analyzed groups was not

Table 3

Surgical characteristics of both study groups.

\begin{tabular}{|c|c|c|c|c|}
\hline & & Group A & Group B & $\mathrm{p}$ Value \\
\hline \multicolumn{2}{|c|}{ Surgical procedure length (minutes) median (range) } & $128(95-195)$ & $100(75-141)$ & 0.002 \\
\hline \multicolumn{2}{|c|}{ Plates and screws 2.0 (number of fractures) } & 30 & 25 & 0.0240 \\
\hline \multicolumn{2}{|c|}{ Reconstructive osteosynthesis plates with 2.3 screws } & - & 6 & \\
\hline \multicolumn{2}{|c|}{ BMACs use } & 30 & - & - \\
\hline \multirow[t]{3}{*}{ Postoperative pain } & Mild & 10 & 8 & 1.000 \\
\hline & Moderate & 7 & 8 & \\
\hline & Severe & - & 2 & \\
\hline \multicolumn{2}{|c|}{ Diffused local-cervical hematoma } & 1 & 5 & 0.1774 \\
\hline \multicolumn{2}{|c|}{ Hospital stay (days) median (range) } & $3(2-7)$ & $4(2-6)$ & 0.2725 \\
\hline
\end{tabular}


statically significant $(p=0.3377$ ), their incidence was higher in patients treated without BMACs than in group A; this could find a reason in the use of a wider surgical incision, implying the exposure of almost all of the mandibular body, to allow the use of large reconstructive osteosynthesis plates, which had been seen associated with a three-time higher incidence of local complications. In this setting, we emphasize that the longer surgical procedure length of group $A$ in comparison to group B could be justified by the need to flatten a learning curve where a new minimally invasive approach requires the use of small skin incisions (shorter than $4.5 \mathrm{~cm}$ ), even in case of multiple, comminuted and displaced fractures, together with the use of plate and screws 2.0 only, and the final addition of regenerative tissue. Nevertheless, the shorter hospital stay recorded in the BMACs group than in group B, together with the lower complication incidence, show the presence of a concrete healing process enhanced by the new surgical protocol.

In our opinion, adding BM-derived progenitor cells can enhance the local concentration of osteoprogenitor cells which are capable of differentiating into osteoblasts and migrating to the bone graft, where they settle, proliferate, differentiate into osteoblasts, and form new bone tissue (Caplan and Bruder, 2001). Yuan et al. (2007) showed in a canine model that critical-sized segmental defects of the canine mandible can be repaired by osteogenically induced BMSCs with a biodegradable b-TCP scaffold. Moreover, the addition of platelet-rich plasma (PRP), which includes various growth factors (Marx et al., 1988) and offers osteoinductive properties (Kanno et al., 2005), contributes to cellular proliferation, matrix formation, collagen synthesis, osteoid production, and other processes that accelerate tissue regeneration. In terms of using these autologous materials, tissue engineering for bone regeneration has been shown to be an attractive alternative to autogenous or synthetic bone substitutes (Ito et al., 2006; Yamada et al., 2004; Buser et al., 1990). It has the additional advantage of using the host as a donor source, which avoids bone graft donor site morbidity. Animal studies have yielded variable results (Gandhi et al., 2006; Gerard et al., 2006; Sarkar et al., 2006). Unfortunately, clinical studies have not seen the same success with the use of PRP as has been seen with other osteoinductive products such as BMPs (Carreon et al., 2005). This lack of success may be due to the relatively low concentration of growth factors that can be extracted in this manner-only about five times the concentration of plasma (Gerard et al., 2006) compared with the 10-fold (Carreon et al., 2005) increase in synthetic BMPs (Westerhuis et al., 2005).

Several key questions related to the techniques of edentulous mandible fracture repair and the uniqueness of the surgical environment remain unanswered (Marciani, 2001). In fact, although it is impossible to ascertain from the recent literature how patients who received more or less invasive procedures would have fared if left untreated or treated to a dysfunctional endpoint because of lack of prospective and large cases studies, from our preliminary results we can advocate a surgical approach that encourages the following:

1) to use a minimally invasive surgical approach in patients over 65 years with co-morbidities who are affected by mandibular atrophy class II or III, even in the case of comminuted and displaced fractures, by avoiding the use of large reconstructive osteosynthesis plates, in order to reduce hospital stay and postoperative complications;

2 ) to always consider co-morbidities to prevent possible wound healing delay and to better address the surgical protocol; in fact, large skin incisions and wide exposure of the mandible should be prevented mainly in patients affected by diabetes and vascular systemic disease;

3) bone bank allograft is strongly recommended in cases of comminuted and displaced fractures for classes II and III of mandibular atrophy, instead of harvesting iliac crest bone graft, which could make surgical operation time much longer than usual, and which is linked to a higher donor site morbidity in elderly patients;

4) a minimally invasive approach should always consist in performing extra-oral open access, with a skin incision length between 3 and $4.5 \mathrm{~cm}$, using osteosynthesis plates and 2.0 screws;

5 ) injection of BMACs sub-periosteally, after osteosynthesis plates have been fixed;

6) to reduce surgical operation length, TEB and PRP could be harvested and prepared simultaneously at the beginning of the operation, by a second surgeon.

\section{Conclusion}

Here, we have demonstrated consistent results from the first-inhuman study for atrophic mandibular bone fracture regeneration using BMACs in combination with a less invasive surgical approach, yielding good immediate results and ensuring functional long-term outcomes in patients with mandibular fractures in atrophy classes II and III. Despite the fact that an early learning curve could affect surgical procedure length $(\mathrm{p}=0.002)$, technical details such as a faster recovery with an overall hospital stay 1 day shorter than usual $(\mathrm{p}=0.2725)$ and a lower incidence of postoperative complications, are worth mentioning and are considered in our final results analysis. Because this study aimed to assess the safety and response to BMAC use with a small but, in our opinion, significant patients group because of the uncommon pathology, we can affirm that this technique provides an easily renewable and reliable source of osteogenic stem cells with numerous advantages when compared with standard open-grafting techniques and related outcomes.

\section{Conflict of interest}

All authors declare that they do not have any financial or personal relationships, any sponsorship or founding arrangements related to this research, with other people or organizations that could inappropriately influence this work. Moreover, all authors also do not have any conflicts of interest.

\section{Financial disclosure}

None of the authors has financial conflicts or interests to report in association with the contents of this paper.

\section{References}

Barber HD: Part I: conservative management of the fractured atrophic edentulous mandible. J Oral Maxillofac Surg 59: 789-791, 2001

Beltrán-Aguilar ED, Barker LD, Canto MT, et al: Centers for disease control and prevention: surveillance for dental caries, dental sealants, tooth retention, edentulism, and enamel fluorosis, United States, 1988-1994 and 1999-2002. Available at: http://www.cdc.gov/mmwr/preview/mmwrhtml/ss5403a1.htm, 2007 [Accessed 16 November 2007]; 2007

Bruce RA, Strachan DS: Fractures of the edentulous mandible: the Chalmers J. Lyons Academy study. J Oral Surg 34: 973, 1976

Bruce RA, Ellis E: The second Chalmers J. Lyons Academy study of fractures of the edentulous mandible. J Oral Maxillofac Surg 51: 904, 1993

Buchbinder D: Treatment of fractures of the edentulous mandible, 1943 to 1993: a review of the literature. J Oral Maxillofac Surg 51: 1174, 1993

Buser D, Brägger U, Lang N, Nyman S: Regeneration and enlargement of jaw bone using guided tissue regeneration. Clin Oral Implant Res 1: 22-32, 1990

Caplan AI, Bruder SP: Mesenchymal stem cells: building blocks for molecular medicine in the 21st century. Trends Mol Med 7: 259-264, 2001

Carreon LY, Glassman SD, Anekstein Y, et al: Platelet gel (AGF) fails to increase fusion rates in instrumented posterolateral fusions. Spine 30: E243-E246, 2005 discussion E247

Clayman L, Rossi E: Fixation of atrophic edentulous mandible fractures by bone plating at the inferior border. J Oral Maxillofac Surg 70: 883-889, 2012

Ellis III E, Price C: Treatment protocol for fractures of the atrophic mandible. J Oral Maxillofac Surg 66: 421-435, 2008 
Eyrich GK, Gratz KW, Sailer HF: Surgical treatment of fractures of the edentulous mandible. J Oral Maxillofac Surg 55: 1081, 1997

Gandhi A, Dumas C, O'Connor JP, et al: The effects of local platelet rich plasma delivery on diabetic fracture healing. Bone 38: 540-546, 2006

Gerard D, Carlson ER, Gotcher JE, et al: Effects of platelet-rich plasma on the healing of autologous bone grafted mandibular defects in dogs. J Oral Maxillofac Surg 64: 443-451, 2006

Herford AS, Boyne PJ: Reconstruction of mandibular continuity defects with bone morphogenic protein-2 (rhBMP-2). J Oral Maxillofac Surg 66: 616-624, 2008

Iatrou I, Samaras C, Lygidakis NT: Miniplate osteosynthesis for fractures of the edentulous mandible: a clinical study, 1989-1996. J Craniomaxillofac Surg 26: 400, 1998

Ide C, Nakai Y, Nakano N, Seo T, Yamada Y, Endo K, et al: Bone marrow stromal cell transplantation for treatment of sub-acute spinal cord injury in rat. Brain Res 1332: $32-47,2010$

Inukai T, Katagiri W, Yoshimi R, Osugi M, Kawai T, Hibi H, et al: Novel application of stem cell-derived factors for periodontal regeneration. Biochem Biophys Res Commun 430: 763-768, 2013

Ito K, Yamada Y, Naiki T, Ueda M: Simultaneous implant placement and bone regeneration around dental implants using tissue-engineered bone with fibrin glue, mesenchymal stem cells (MSCs), and platelet-rich plasma (PRP). Clin Oral Implant Res 17: 579-586, 2006

Kan I, Melamed E, Offen D: Integral therapeutic potential of bone marrow mesenchymal stem cells. Curr Drug Targets 6: 31-41, 2005

Kanno T, Takahashi T, Tsujisawa T, et al: Platelet-rich plasma enhances human osteoblast-like cell proliferation and differentiation. J Oral Maxillofac Surg 63: 362-369, 2005

Katagiri W, Osugi M, Kawai T, Ueda M: Novel cell-free regenerative medicine of bone using stem cell derived factors. Int J Oral Maxillofac Implant 28: 1009-1016, 2013

Katagiri W, Osugi M, Kawai T, Hibi H: First-in-human study and clinical case reports of the alveolar bone regeneration with the secretome from human mesenchymal stem cells. Head Face Med 12: 5, 2016

Kawai T, Katagiri W, Osugi M, Sugimura Y, Hibi H, Ueda M: Secretomes from bone marrow-derived mesenchymal stromal cells enhance periodontal tissue regeneration. Cytotherapy 17: 369-381, 2015

Kunz C, Hammer B, Prein J: [Fractures of the edentulous atrophic mandible: management and complications] [in German]. Mund Kiefer Gesichtschir 5: 227, 2001

Langer R, Vacanti JP: Tissue engineering. Science 260: 920-926, 1993

Luhr HG, Reidick T, Merten HA: Results of treatment of fractures of the atrophic edentulous mandible by compression plating: a retrospective evaluation of 84 consecutive cases. J Oral Maxillofac Surg 54: 250-254, 1996

Marciani RD: Part II: invasive management of the fractured atrophic edentulous mandible. J Oral Maxillofac Surg 59: 792-795, 2001

Marx RE, Carlson ER, Eichstaedt RM, Schimmele SR, Strauss JE, Georgeff KR: Platelet-rich plasma, growth factor enhancement for bone grafts. Oral Surg Oral Med Oral Pathol Oral Radiol Endod 85: 638-646, 1988

Muller-Ehmsen J, Whittaker P, Kloner RA, Dow JS, Sakoda T, Long TI, et al: Survival and development of neonatal rat cardiomyocytes transplanted into adult myocardium. J Mol Cell Cardiol 34: 107-116, 2002
Ogata K, Katagiri W, Osugi M, Kawai T, Sugimura Y, Hibi H, et al: Evaluation of the therapeutic effects of conditioned media from mesenchymal stem cells in a rat bisphosphonate-related osteonecrosis of the jaw-like model. Bone 74: 95-105, 2015

Osugi M, Katagiri W, Yoshimi R, Inukai T, Hibi H, Ueda M: Conditioned media from mesenchymal stem cells enhanced bone regeneration in rat calvarial bone defects. Tissue Eng Part A 18: 14779-21489, 2012

Prins HJ, Rozemuller H, Vonk-Griffioen S, Verweij VG, Dhert WJ, SlaperCortenbach IC, et al: Bone-forming capacity of mesenchymal stromal cells when cultured in the presence of human platelet lysate as substrate for fetal bovine serum. Tissue Eng A 15: 3741-3752, 2009

Rickert D, Sauerbier S, Nagursky H, Menne D, Vissink A, Raghoebar GM: Maxillary sinus floor elevation with bovine bone mineral combined with either autogenous bone or autogenous stem cells: a prospective randomized clinical trial. Clin Oral Implant Res 22: 251-258, 2011

Sarkar MR, Augat P, Shefelbine SJ, et al: Bone formation in a long bone defect model using a platelet-rich plasma-loaded collagen scaffold. Biomaterials 27: 1817-1823, 2006

Sen MK, Miclau T: Autologous iliac crest bone graft: should it still be the gold standard for treating nonunions? Inj Int J Care Inj 38(S1): S75-S80, 2007

St John TA, Vaccaro AR, Sah AP, et al: Physical and monetary costs associated with autogenous bone graft harvesting. Am J Orthop 32: 18-23, 2003

Toma C, Wagner WR, Bowry S, Schwartz A, Villanueva F: Fate of culture-expanded mesenchymal stem cells in the microvasculature: in vivo observations of cell kinetics. Circ Res 104: 398-402, 2009

Watson L, Elliman SJ, Coleman CM: From isolation to implantation: a concise review of mesenchymal stem cell therapy in bone fracture repair. Stem Cell Res Ther 5: 51, 2014

Westerhuis RJ, van Bezooijen RL, Kloen P: Use of bone morphogenetic proteins in traumatology. Injury 36: 1405-1412, 2005

Wittwer G, Adeyemo WL, Turbani D, et al: Fractures based on the degree of atrophy: experience with different plating systems. A retrospective study. J Oral Maxillofac Surg 64: 230, 2006

Wongchuensoontorn C, Liebehenchel N, Schwarz U, Schmelzeisen R, Gutwald R, Ellis III E, et al: Application of a new chair-side method for the harvest of mesenchymal stem cells in a patient with nonunion of a fracture of the atrophic mandible. A case report. JCMS 37: 155-161, 2009

Yamada Y, Hara K, Nakamura S, Ueda M, Ito K, Nagasaka T: Minimally invasive approach with tissue engineering for severe alveolar bone atrophy case. Int J Oral Maxillofac Surg 42: 260-262, 2013

Yamada Y, Ueda M, Naiki T, Takahashi M, Hata K, Nagasaka T: Autogenous injectable bone for regeneration with mesenchymal stem cells (MSCs) and platelet-rich plasma (PRP): tissue-engineered bone regeneration. Tissue Eng 10: 955-964, 2004

Yamamoto K, Matsusue Y, Murakami K, Horita S, Sugiura T, Kirita T: Maxillofacial fractures in older patients. J Oral Maxillofac Surg 69: 2204-2210, 2011

Yuan J, Cui L, Zhang WJ, Liu W, Cao Y: Repair of canine mandibular bone defects with bone marrow stromal cells and porous beta-tricalcium phosphate. Biomaterials 28: 1005-1013, 2007 